\title{
Novel Directional Nanoantennas for Single-Emitter Sources and Wireless Nano-Links
}

\author{
Maciej Klemm \\ Department of Electrical and Electronic Engineering, University of Bristol, Woodland Road, Bristol BS1 8UB, UK \\ Correspondence should be addressed to Maciej Klemm, m.klemm@bristol.ac.uk
}

Received 30 September 2011; Revised 19 December 2011; Accepted 20 December 2011

Academic Editor: Nicolas Bonod

Copyright () 2012 Maciej Klemm. This is an open access article distributed under the Creative Commons Attribution License, which permits unrestricted use, distribution, and reproduction in any medium, provided the original work is properly cited.

Optical nanoantennas are emerging as one of the key components in the future nanophotonic and plasmonic circuits. The first optical nanoantennas were in a form of simple spherical nanoparticles. Recently more complex Yagi-Uda nanoantenna structures were demonstrated. These nanoantennas enhance radiation of single emitters and provide well-defined directional radiation. In this contribution, we present the novel design of the directional nanoantenna, which is excited from the propagating mode of the plasmonic waveguide. The nanoantenna design is based on the travelling wave principle, well known at RF/microwave frequencies. By properly designing the propagating parts of the nanoantenna, a very efficient coupling to free space wave impedance can be achieved. Furthermore, the control over the radiation direction and beam width is relatively easy with this nanoantenna. Compared to the previously published Yagi-Uda designs, the new nanoantenna presented in this work has directivity three times higher.

\section{Introduction}

In the future we foresee the development of optical wireless communication networks at the nanoscale [1]. In this work we will focus on one specific problem in the development of the physical layer of such wireless networks-design of novel nanoantennas (often also referred to as optical antennas, or plasmonic antennas in the open literature). Nanoantennas are key components and enabling technology for the future wireless connectivity at the nanoscale, coupling light from external world to nanoscale circuits and vice versa.

Nanoantennas have their origins in the field of physics and more precisely in near-field microscopy. Wessel in 1985 was the first to mention explicitly the analogy of local microscopic light sources to classical antennas [2] - a concept that has since been thoroughly explored. History of nanoantennas can be found in [3].

As one of the main applications for novel nanoantennas we envisage (a) on-chip and intrachip wireless nano-links in future micro- and nanodevices and (b) single-emitter sources coupled to the directional nanoantenna.

The coupling of the nanoantenna to single-emitter light sources has been investigated by several groups theoretically
[4-7] as well as experimentally [8, 9]. Nanoantenna in this application is used mainly to direct and focus light. Due to the lack of true nanoscale light sources, the coupling of the emitter (e.g., quantum dot) to the nanoantenna has been achieved by proximity coupling (near-field coupling) which is rather inefficient. Ideally, one would like to connect the source and the nanoantennas by efficient transmission line (waveguide), similarly as at RF and microwave frequencies. When designing nanoantenna, it is important to consider not only the radiating part but also the feeding arrangement.

Nanoantenna-enabled wireless nano-links could be considered as an alternative solution to the problem of an interconnect bottleneck in the next generation-integrated circuits (ICs). Currently silicon photonics is being actively researched in academia and by many electronic manufacturers, who see it as a means for keeping up with Moor's law by using optical interconnects to provide faster data transfers between and within microchips. However, size of dielectric waveguides used in silicon photonics is fundamentally limited by diffraction optics, posing a limit on the maximum achievable density of such interconnect technology. To tackle this problem, plasmonic integrated circuits have been most recently proposed [10] to achieve true nanoscale integration 


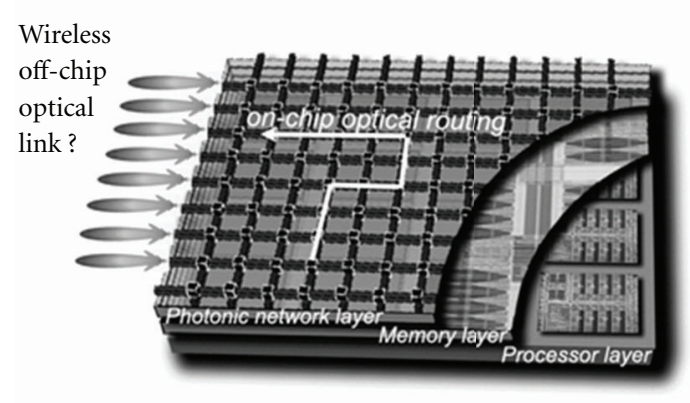

FIGURE 1: A concept of using directional nanoantennas as means of providing wireless on-chip and intrachip communication. Photo is modified from IBM.

in future ICs. Unfortunately, the price to pay for extremely high density of plasmonic interconnects is relatively high loss in such waveguides (compared to silicon photonics). We would like to propose another approach to the optical interconnect problem-wireless connectivity using nanoantennas. We can imagine a very high data rate chip-to-chip and intrachip links supported mainly by nanoantennas with very few interconnects needed. Moreover, besides providing a "simple" on-/intrachip connections, nanoantennas could offer an advantage of having an extra wireless functionality in future integrated circuits.

\section{Differences between RF/Microwave Antennas and Plasmonic Nanoantennas}

Theory of RF/microwave antennas was being developed for well over 100 years. Several design rules for antenna engineers exist, and probably tens of textbooks are at their disposal. However, if one wishes to design a nanoantenna, not even a single book on a subject is available. Unfortunately, due to different underlying physics at optical wavelengths, based on plasmon resonance (specific electromagnetic solution of Maxwell's equations at the metaldielectric interface at optical wavelengths [11]), most design rules for RF/microwave antennas cannot be simply used in nanoantennas. Even for the simplest possible antenna, a dipole, there is a significant difference in radiation patterns of the plasmonic nanoantenna and the RF antenna [12], as shown in Figure 2.

\section{Nanoantenna Design}

The design of the new directional nanoantenna was performed using commercial electromagnetic finite integration technique (FIT) solver-CST Microwave Studio [13]. In all studies antennas were assumed to be made of gold. We used gold permittivity values as measured by Johnson and Christy [14], which were fitted as the fourth-order polynomial in the numerical solver. Comparison between values from [14] and those used in simulations is shown in Figure 3.
For the proposed new directional nanoantenna, shown in Figure 4, we have chosen a design based on the travellingwave principle [15]. This nanoantenna can be seen as a hybrid between a well-known V-antenna and Vivaldiantenna. In the study we assumed free-space operation only.

The first directional nanoantennas were based on the Uda-Yagi design. However unlike at the RF/microwave frequencies, these nanoantennas were excited either by nearfield coupling of the dipole source (quantum dot in [8]) or by far-field laser radiation [16]. Obviously for our targeted application, integrated on-chip plasmonic circuits, it is desired to excite antennas using a waveguide. As shown in Figure 4(a), our proposed design is easy to integrate with the traditional metal-insulator-metal (MIM) plasmonic waveguide. We used the same MIM waveguide as presented in [17], composed of two $50 \mathrm{~nm}$ by $50 \mathrm{~nm}$ gold stripes, with the gap of $20 \mathrm{~nm}$. The calculated impedance is $449 \Omega$. The complex effective index of the feeding plasmonic waveguide is $n_{\text {eff }}=2.19+i 0.068$.

The leaky nature of propagating plasmons in the proposed design makes the operation of the nanoantenna similar to the $\mathrm{V}$-antenna (composed of two wires over a ground plane). The main difference between RF and nanoversion is that in plasmonics the ground plane is not used (due to the very nature of the plasmonic mode), and the concept of the termination (used in the RF V-antenna) does not exist at the nanoscale. For these two reasons the proposed nanoantenna can be seen being close to another travelling wave RF design-Vivaldi antenna.

During the design of our nanoantennas we did not aim at any particular wavelength. Results presented hereinafter are for the wavelength of $780 \mathrm{~nm}$. However, this nanoantenna is fairly broadband in its operation and can be used at other wavelengths. The major difference in operation will be due to the dispersive permittivity of gold.

In Figure 5(a) we present the radiation pattern for the designed nanoantennas at $780 \mathrm{~nm}$. The calculated radiation efficiency at this wavelength is $48 \%$. Considering a lossy nature of plasmonic structures this is a relatively good result (it also includes losses in the feeding waveguide).

The most important result of the new design is a high maximum directivity value of around 24 (linear scale). This is a major improvement over previously designed Yagi-Uda nanoantennas. To obtain a more quantitative comparison we have simulated gold Yagi-Uda nanoantenna (shown in Figure 5(b)) similar to this presented in [18]. We could therefore compare only radiation patterns and not efficiencies between these two designs. As clearly seen in Figure 5 the nanoantenna proposed in this work has a maximum directivity of 24, more than three times higher directivity achieved by Yagi-Uda (around 7).

When analysing results for traditional $\mathrm{RF} /$ microwave antennas, the input matching (or return loss) is often considered as one of the main performance measures. At the nanoscale however, although the concept of nanoantenna impedance has been investigated $[19,20]$, because of the lack of integrated nanoscale sources the input matching is not being considered at the moment. By "integrated nanoscale sources" we mean the sources similar to those found at 


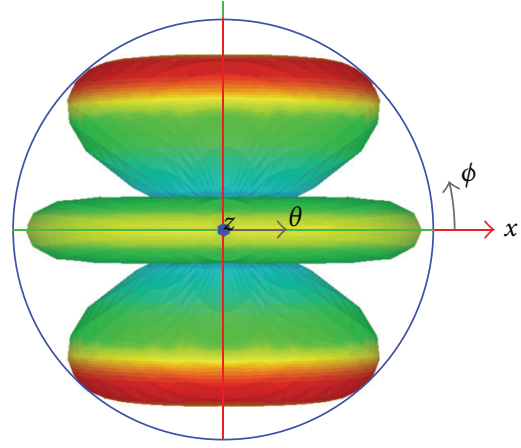

(a)

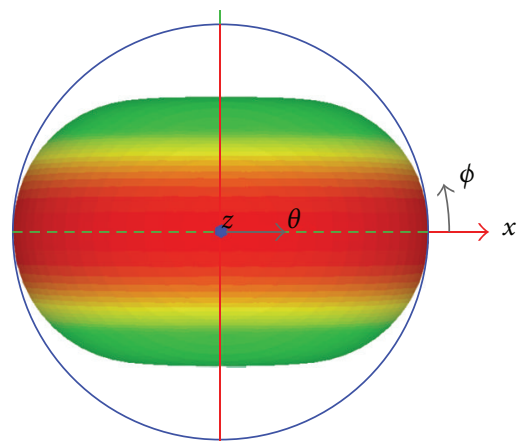

(b)

FIGURE 2: Radiation pattern of dipole antenna in third-order resonance: (a) standard RF dipole and (b) nanoantenna.

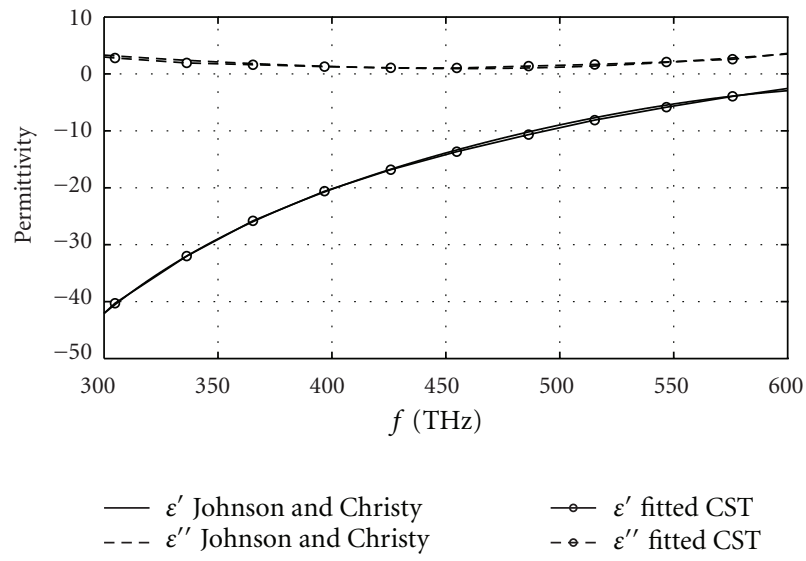

Figure 3: Complex permittivity of gold used in EM simulations (CST Microwave Office). Values are based on measured data from [14] and fitted using fourth-order polynomial.

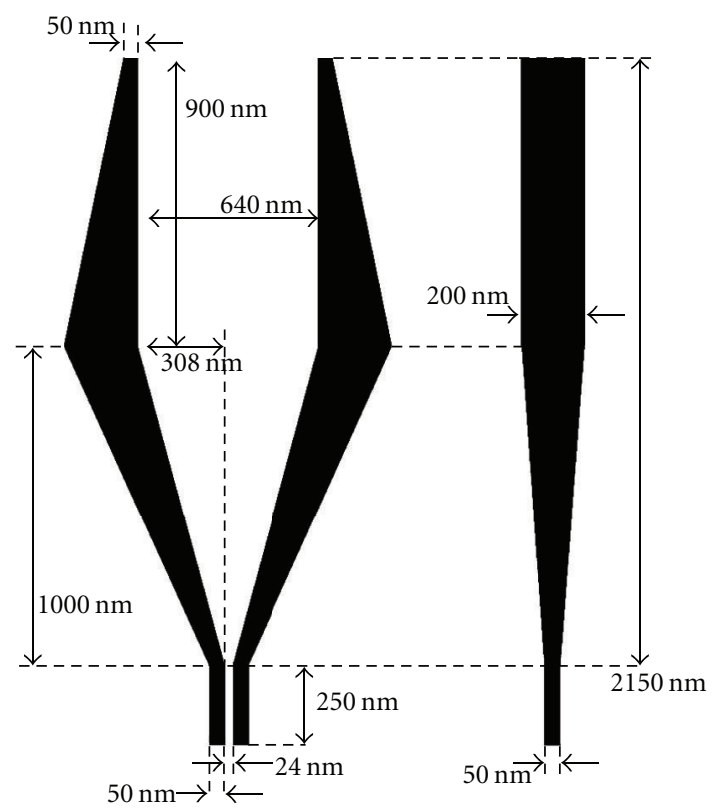

FIGURE 4: Geometry of the proposed new directional nanoantenna: top view (left) and side view (right).
RF/microwave frequencies, which can be simply "plugged" to the antenna or transmission line. Also a concept of "standard" impedance (usually $50 \Omega$ for RF/microwave circuits and antennas) is currently not defined for nanocircuits.

\section{Nanoantenna Coupled to the Single Emitter Source}

Directional nanoantennas could also be used for extracting light from single photon sources. This was recently demonstrated in [8], where Yagi-Uda nanoantenna was used to direct light from the quantum dot (QD). Because both the QD and nanoantenna were placed on the surface of the highindex material, main radiation was directed in the substrate. In Figure 6 we present the example of our new directional nanoantennas coupled to the dipolar emitter as well as the reference result of the stand-alone dipole emitter embedded in high-index material. Dipole is oriented along the $x$-axis (to excite the plasmonic waveguide) and placed $2.15 \mu \mathrm{m}$ below the surface of the material with $n=1.5$. This distance was dictated solely by the size of the nanoantenna. Figures 6(c) and 6(d) present the electric field produced by the stand-alone and nanoantenna-coupled dipole, respectively. Due to the reflection off the interface between materials, most radiation goes into the high index substrate for the single dipole. The calculated far-field radiation for this case is shown in Figure 6(e). However, unidirectional emission was achieved when the dipole was coupled to the new directional nanoantenna. The light generated by the dipole is propagated along the plasmonic structure and efficiently radiated into the low-index material (free space). The radiation pattern of the dipole-nanoantenna-coupled system is shown in Figure 6(f). Most importantly, in the direction of the main radiation beam the amplitude of the electric field is more than $30 \mathrm{~dB}$ higher when the dipole emitter is coupled to the nanoantenna. Exact electric field (Ex) values taken $1 \mu \mathrm{m}$ above the interface between materials (see Figure 6(b) for exact location of the E-field probe) are presented in Figure 7.

Naturally, the nanoantenna embedded in the high-index material (Figure 6(b)) and its directional radiation into the free space (Figures 6(d) and 6(f)) could be used to provide wireless connectivity in the intrachip and board-to-board 


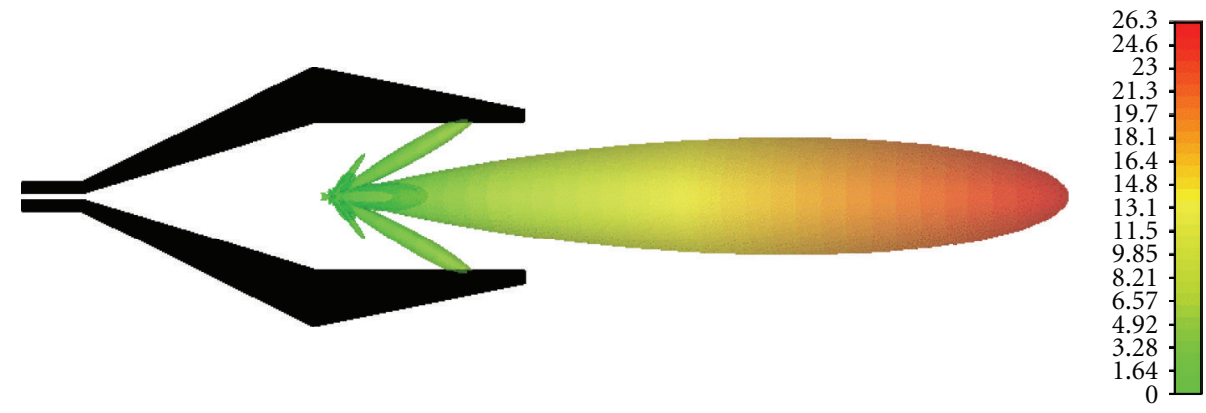

(a)

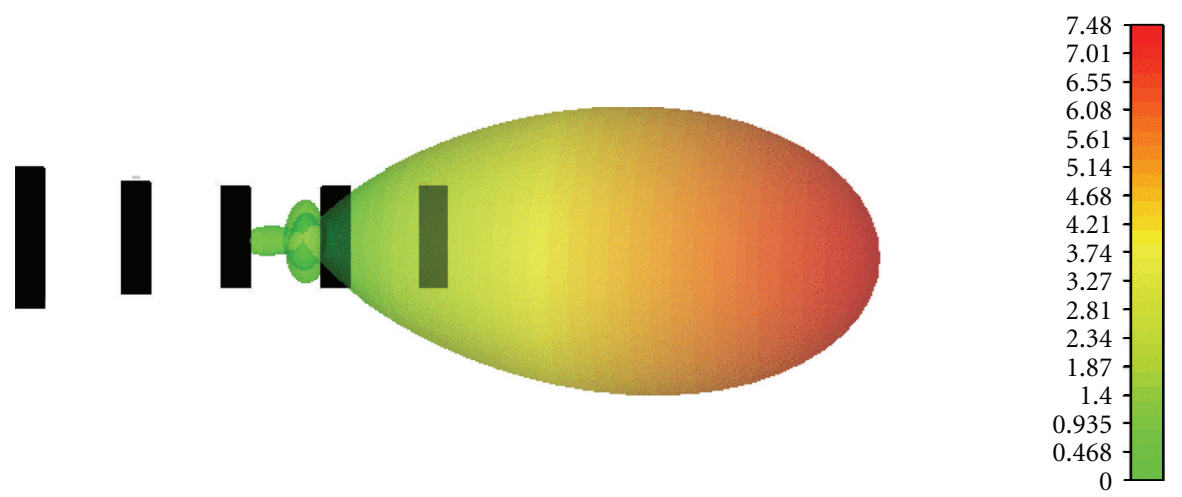

(b)

FIgURE 5: Comparison of simulated directivity (linear scale) far-field radiation patterns for (a) new directional nanoantennas designed in this work, and (b) Yagi-Uda type nanoantennas from [18]. Both nanoantennas are shown in top view.

optical links (as shown in, e.g., Figure 1). Moreover, we believe that in future 3D-integrated circuits with optical interconnects, the presented directional nanoantenna could be used as an "optical via", providing vertical connection between different chip layers. Currently proposed solutions of $3 \mathrm{D}$ optical integration involve microlenses and micromirrors [21].

\section{Nanoantennas for Wireless Optical Nano-links}

In the future we foresee the development of optical wireless communication networks at the nanoscale. Nanoantennas are key components and an enabling technology for future optical wireless connectivity at the nanoscale, coupling light from external world to nanoscale circuits and vice versa. As one of the main applications for novel nanoantennas we envisage a provision of on-chip and intrachip wireless nanolinks in future micro- and nanodevices.

One of the main challenges in using nanoantennas fabricated on high-index substrates is the problem with most of the radiated power going into the substrate [8]. This is presented in Figure 8 for the nanolink composed of our new nanoantennas on glass. The distance between antennas is $4 \mu \mathrm{m}$. Figure 8 shows the amplitude of electric field as radiated by the transmitting antenna on the left-hand side. Most of the radiation goes straight into the glass, which is obviously not desired in achieving efficient transmission between antenna.
To overcome the problem of radiation to the substrate, we have embedded our nanoantennas in high-index material and placed on the edge of the substrate, as shown in Figure 9. The distance between antennas is again $4 \mu \mathrm{m}$ and the medium between them is free space. Practically, this scenario could be realized in the case of end-fire onchip nanoantennas for interchip interconnects (see, e.g., Figure 1). In this case we can observe symmetrical radiation with the maximum in the direction of the receiving antenna on the right-hand side, where we can clearly see the propagation of the guided wave along the nanoantenna.

The last example of the nanolink is presented in Figure 10 where both nanoantennas as again embedded in the highindex material, which is also acting as the propagation medium. This scenario could practically be considered in multilayered integrated circuits, where nanoantennas could in-plane connectivity, as well as connections between layers on multiple chip levels. As expected, because of the homogeneous environment the nanoantenna radiates horizontally towards the receiving antenna. This is an efficient wireless link.

The quantitative comparison of transmission efficiency of all three nano-links is shown in Figure 11. We compare the magnitude of the scattering parameter S21, indicating the power captured by the receiving antenna. At $300 \mathrm{THz}$ we can see that the best transmission is achieved using the link from Figure 10, with both antennas embedded in glass. Slightly less efficient is the end-fire-like nano-link shown in Figure 9. The worst result is achieved with nanoantennas 


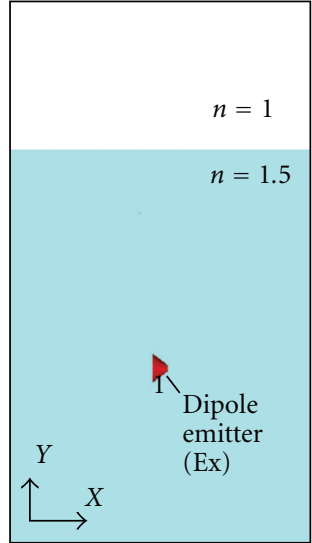

(a)

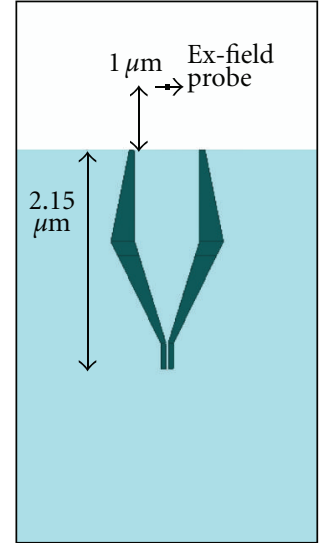

(b)

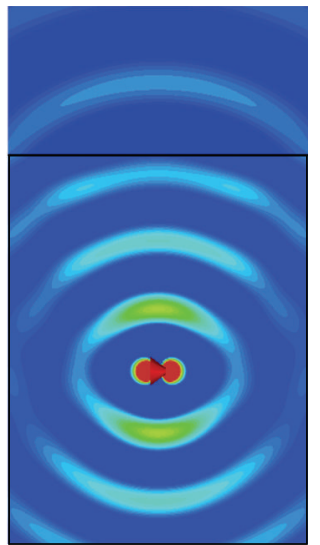

(c)

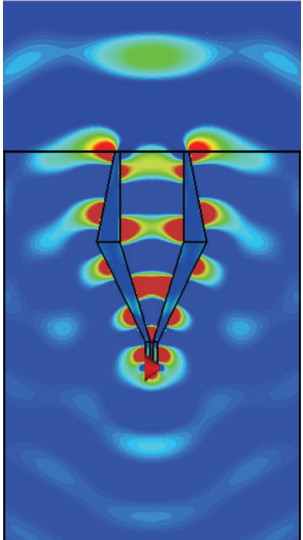

(d)

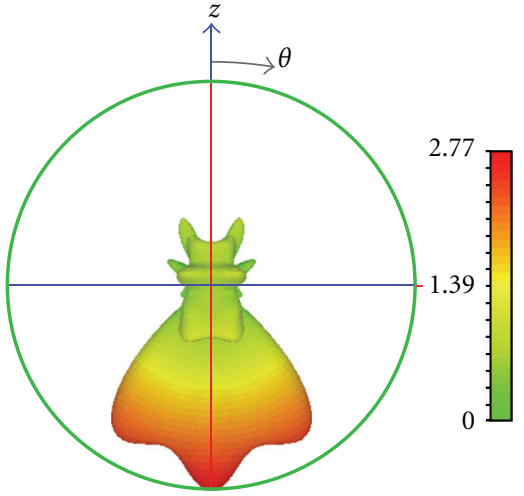

(e)

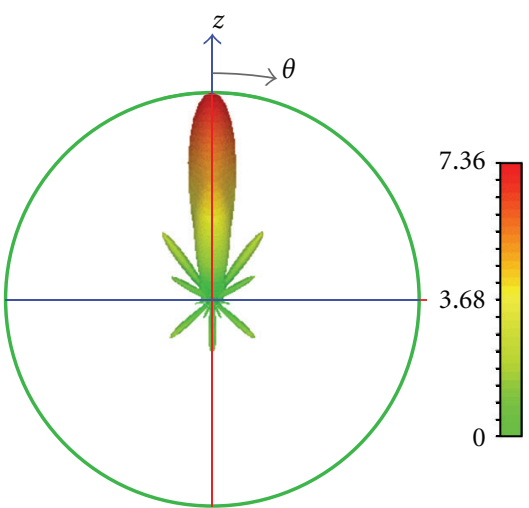

(f)

Figure 6: Comparison of far-field radiation of the dipole emitter embedded in high-index material with and without the nanoantenna. (a) Dipole emitted embedded $2.15 \mu \mathrm{m}$ below the surface of $n=1.5$ material, (b) dipole coupled to the nanoantenna, (c) E-field of the dipole emitter, (d) E-field of the coupled dipole-nanoantenna system, (e) far-field radiation pattern of the dipole in high-index material, and (f) far-field of the dipole coupled to the directional nanoantenna. All results are presented at the wavelength $1 \mu \mathrm{m}$.

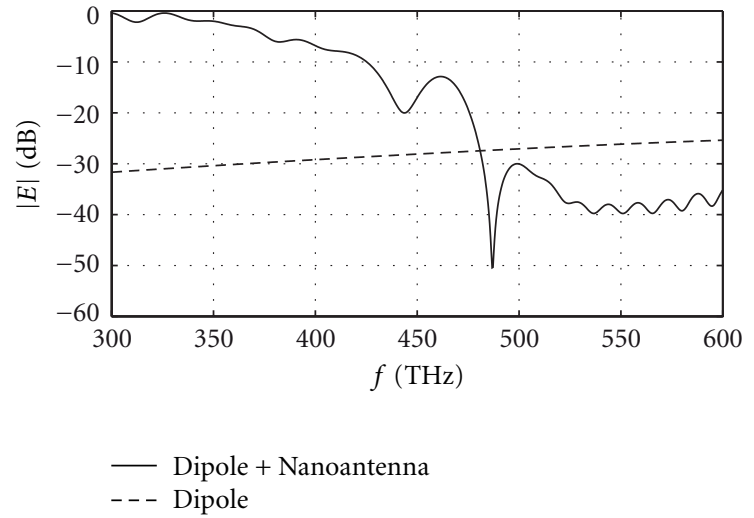

FIGURE 7: Comparison of the amplitude of the radiated Ex electric field taken $1 \mathrm{~mm}$ above the material interface (see Figure 6(b)).

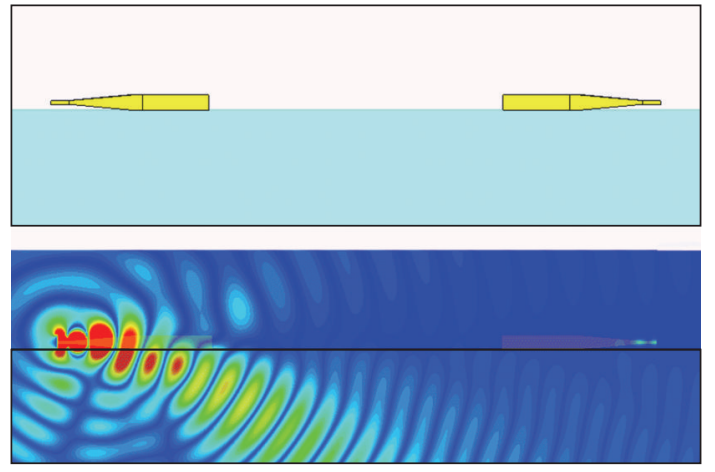

Figure 8: Optical wireless link between nanoantennas on glass substrate and corresponding radiated E-field. Distance between nanoantennas is $4 \mu \mathrm{m}$; frequency is $300 \mathrm{THz}$. placed on glass (Figure 8 ), with the $|\mathrm{S} 21|$ smaller by more than $20 \mathrm{~dB}$, compared with the other two cases. These results clearly show that for most efficient transmission nanoantennas should be placed in homogeneous dielectric on top (superstrate) and below.

\section{Fabrication of Nanoantennas}

After very encouraging design results of the new nanoantennas, we made the first attempts at fabricating them. In Figure 12 we can see two examples of different nanoantenna 


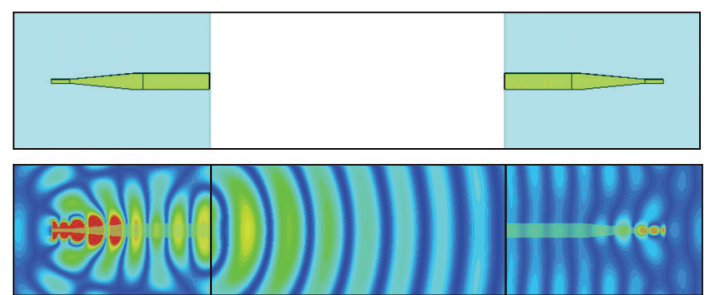

Figure 9: Optical wireless link between nanoantennas embedded in glass substrate and corresponding radiated E-field. Distance between nanoantennas is $4 \mu \mathrm{m}$, frequency is $300 \mathrm{THz}$, and propagation medium is free space.
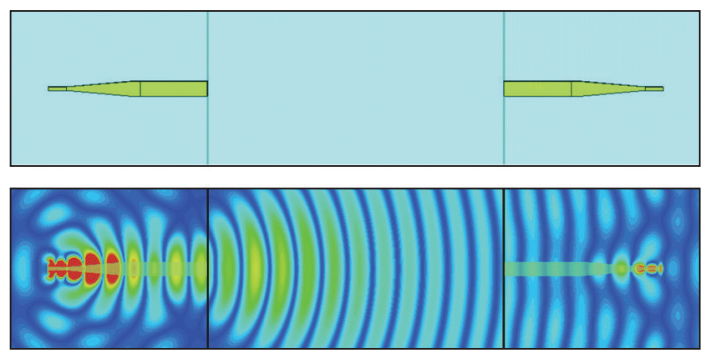

FIGURE 10: Optical wireless link between nanoantennas embedded in glass substrate and corresponding radiated E-field. Distance between nanoantennas is $4 \mu \mathrm{m}$, frequency is $300 \mathrm{THz}$, and propagation medium is glass.

samples. They were fabricated in $50 \mathrm{~nm}$ gold deposited on thick glass slides using focused ion beam milling.

We are currently in a phase of improving our fabrication process and building a measurement setup. All final nanoantenna designs will need to be optimized for operation on a glass substrate.

\section{Conclusions}

In this contribution we presented the novel design of the directional nanoantenna, which is excited from the MIM plasmonic waveguide. The nanoantenna design is based on the travelling wave principle and can be considered as a hybrid between a well-known V-antenna and Vivaldiantenna. The most important result of the new design is a high maximum directivity value of around 24 (linear scale), more than three times higher than that achieved by previously published Yagi-Uda (directivity of 7). First samples of designed nanoantennas were fabricated and measurements are currently being set up. This novel nanoantenna could be used in the end-fire configuration in the intrachip and board-to-board free space optical links or in efficient directional extraction of light emitted by singlephoton sourced.

\section{Acknowledgment}

The authors acknowledge the support of the UK's Engineering and Physical Sciences Research Council (EPSRC),

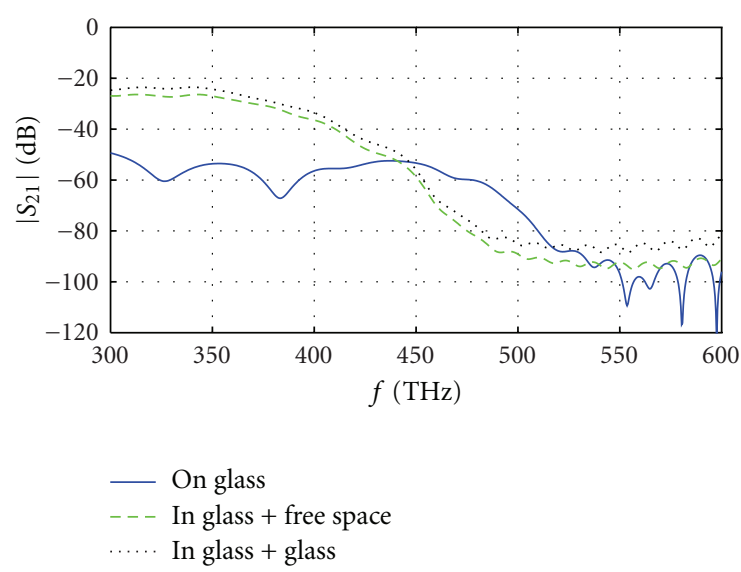

FIgURE 11: Transmission coefficient $|\mathrm{S} 21|$ for three different scenarios of the optical wireless links from Figures 8-10. Distance between nanoantennas is $4 \mu \mathrm{m}$.

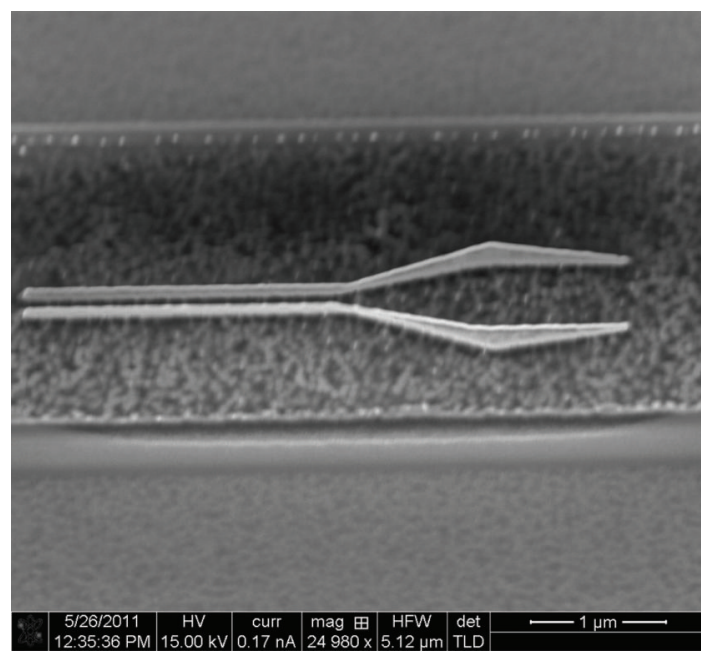

(a)

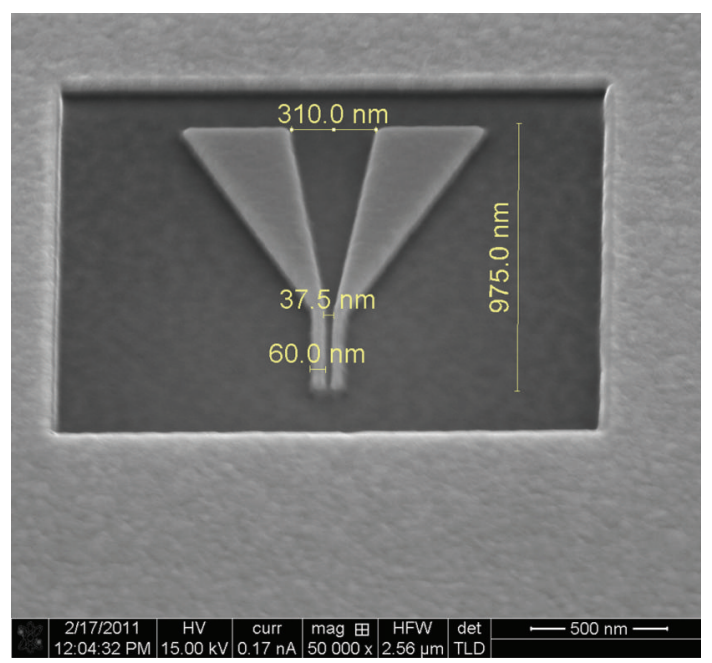

(b)

FIgURE 12: Example of gold nanoantennas fabricated using dual beam focused ion beam (FIB) milling. Nanoantennas were fabricated in $50 \mathrm{~nm}$ gold on thick glass slides. 
Cross-Disciplinary Interfaces Programme (C-DIP) Fellowship Fund, Grant EP/I017852/1.

\section{References}

[1] PhotonicsRoadmapSME (EU FP7 project), "Photonics in ICT: A Technology Roadmap for SMEs on new photonic devices, materials and fabrication technologies," technology roadmap, http://www.photonicroad.eu/upload/PhotonicRoadSME_ Technology\%20Roadmap\%20on\%20Photonics\%20in $\% 20$ ICT.pdf.

[2] J. Wessel, "Surface-enhanced optical microscopy," Journal of the Optical Society of America B, vol. 2, pp. 1538-1540, 1985.

[3] P. Bharadwaj, B. Deutsch, and L. Novotny, "Optical antennas," Advances in Optics and Photonics, vol. 1, pp. 438-483, 2009.

[4] T. H. Taminiau, F. D. Stefani, and N. F. Van Hulst, "Optical nanorod antennas modeled as cavities for dipolar emitters: evolution of sub- and super-radiant modes," Nano Letters, vol. 11, no. 3, pp. 1020-1024, 2011.

[5] B. Stout, A. Devilez, B. Rolly, and N. Bonod, "Multipole methods for nanoantennas design: applications to Yagi-Uda configurations," Journal of the Optical Society of America B, vol. 28, no. 5, pp. 1213-1223, 2011.

[6] K. C. Y. Huang, Y. C. Jun, M.-K. Seo, and M. L. Brongersma, "Power flow from a dipole emitter near an optical antenna," Optics Express, vol. 19, no. 20, pp. 19084-19092, 2011.

[7] V. Giannini, J. A. Sánchez-Gil, O. L. Muskens, and J. G. Rivas, "Electrodynamic calculations of spontaneous emission coupled to metal nanostructures of arbitrary shape: nanoantenna-enhanced fluorescence," Journal of the Optical Society of America B, vol. 26, no. 8, pp. 1569-1577, 2009.

[8] A. G. Curto, G. Volpe, T. H. Taminiau, M. P. Kreuzer, R. Quidant, and N. F. Van Hulst, "Unidirectional emission of a quantum dot coupled to a nanoantenna," Science, vol. 329, no. 5994, pp. 930-933, 2010.

[9] Y. Alaverdyan, N. Vamivakas, J. Barnes, C. Lebouteiller, J. Hare, and M. Atatüre, "Spectral tunability of a plasmonic antenna with a dielectric nanocrystal," Optics Express, vol. 19, no. 19, pp. 18175-18181, 2011.

[10] S. A. Maier, M. L. Brongersma, P. G. Kik, S. Meltzer, A. A. G. Requicha, and H. A. Atwater, "Plasmonics-a route to nanoscale optical devices," Advanced Materials, vol. 13, no. 19, pp. 1501-1505, 2001.

[11] S. Maier, Plasmonics: Fundamentals and Applications, Springer, Berlin, Germany, 2007.

[12] J. Dorfmüller, R. Vogelgesang, W. Khunsin, C. Rockstuhl, C. Etrich, and K. Kern, "Plasmonic nanowire antennas: experiment, simulation, and theory," Nano Letters, vol. 10, no. 9, pp. 3596-3603, 2010.

[13] http://www.cst.com/.

[14] P. B. Johnson and R. W. Christy, "Optical constants of the noble metals," Physical Review B, vol. 6, no. 12, pp. 4370-4379, 1972.

[15] C. Balanis, Antenna Theory: Analysis and Design, John Wiley \& Sons, New York, NY, USA, 2005.

[16] T. Kosako, Y. Kadoya, and H. F. Hofmann, "Directional control of light by a nano-optical Yagi-Uda antenna," Nature Photonics, vol. 4, no. 5, pp. 312-315, 2010.

[17] A. Alù and N. Engheta, "Wireless at the nanoscale: optical interconnects using matched nanoantennas," Physical Review Letters, vol. 104, no. 21, Article ID 213902, 2010.

[18] T. H. Taminiau, F. D. Stefani, and N. F. Van Hulst, "Enhanced directional excitation and emission of single emitters by a nano-optical Yagi-Uda antenna," Optics Express, vol. 16, no. 14, pp. 10858-10866, 2008.

[19] A. Alù and N. Engheta, "Input impedance, nanocircuit loading, and radiation tuning of optical nanoantennas," Physical Review Letters, vol. 101, no. 4, Article ID 043901, 2008.

[20] J. J. Greffet, M. Laroche, and F. Marquier, "Impedance of a nanoantenna and a single quantum emitter," Physical Review Letters, vol. 105, no. 11, Article ID 117701, 2010.

[21] B. Ciftcioglu, R. Berman, J. Zhang et al., "A 3-D integrated intrachip free-space optical interconnect for many-core chips," IEEE Photonics Technology Letters, vol. 23, no. 3, pp. 164-166, 2011. 

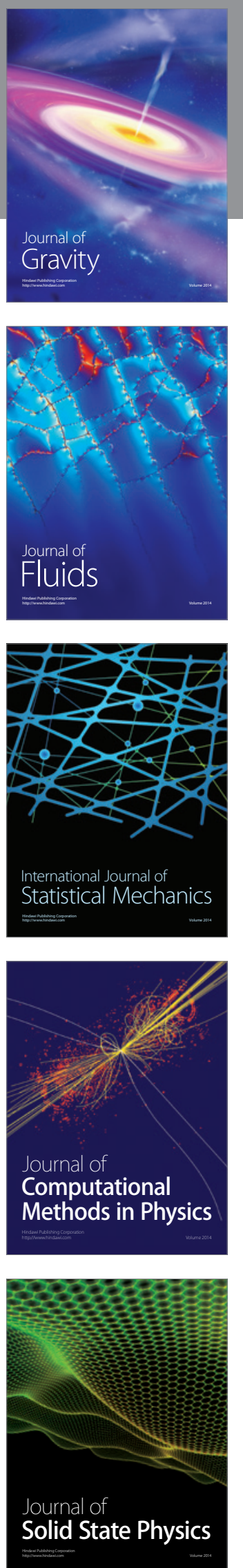

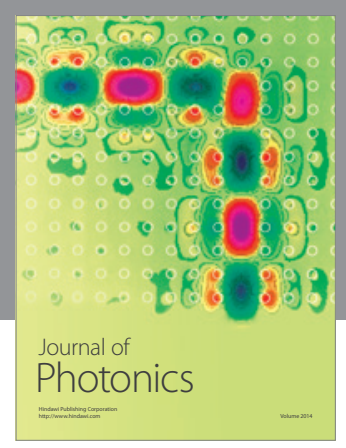

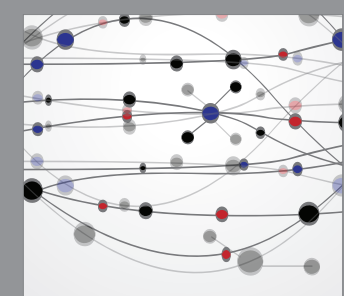

The Scientific World Journal
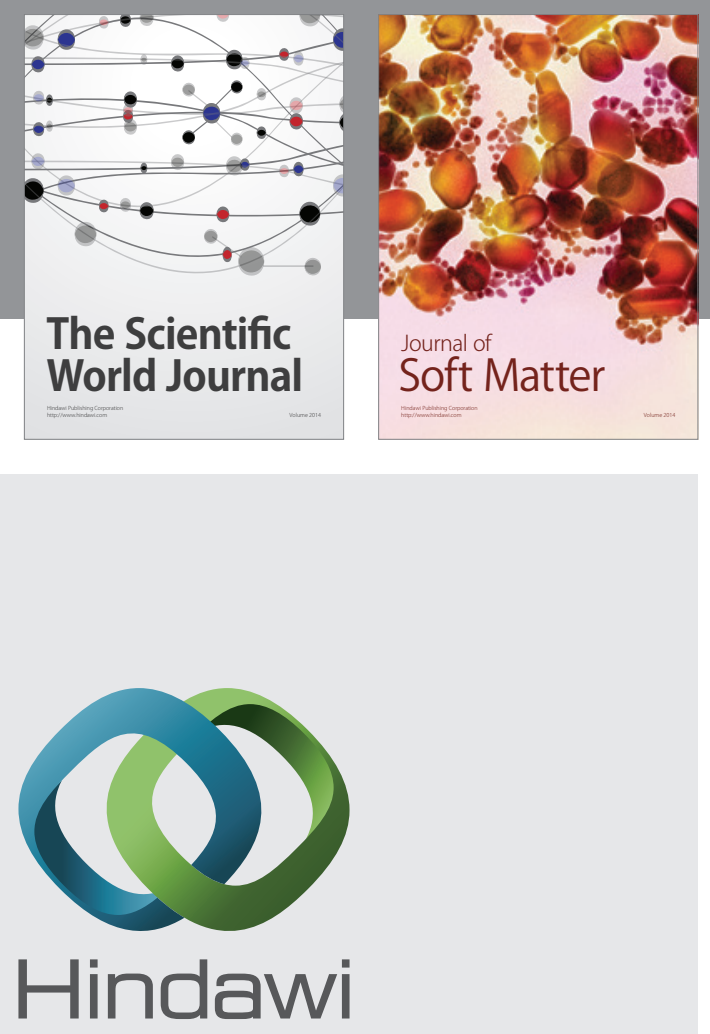

Submit your manuscripts at

http://www.hindawi.com
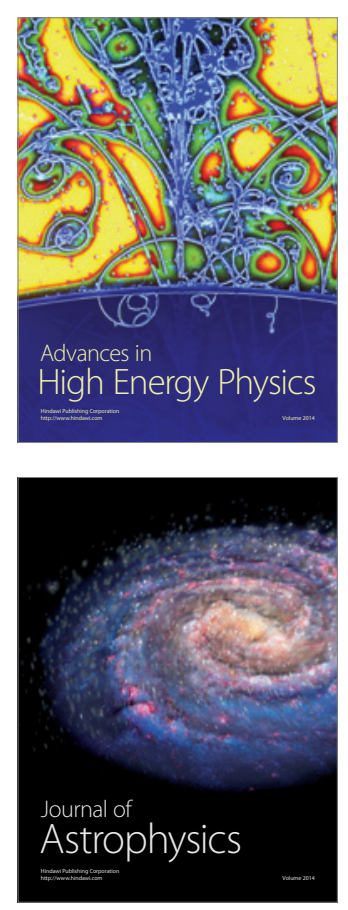
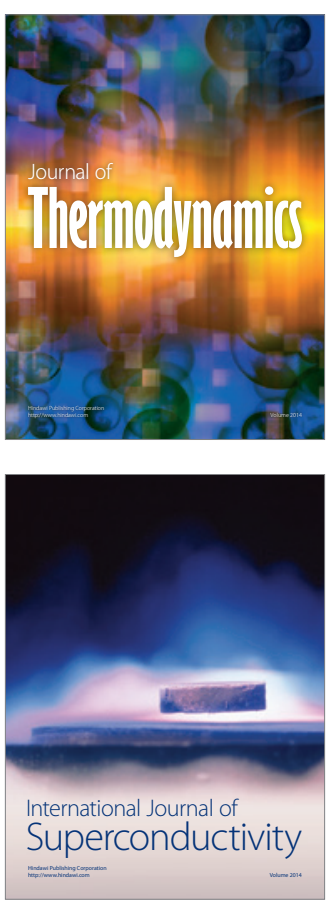
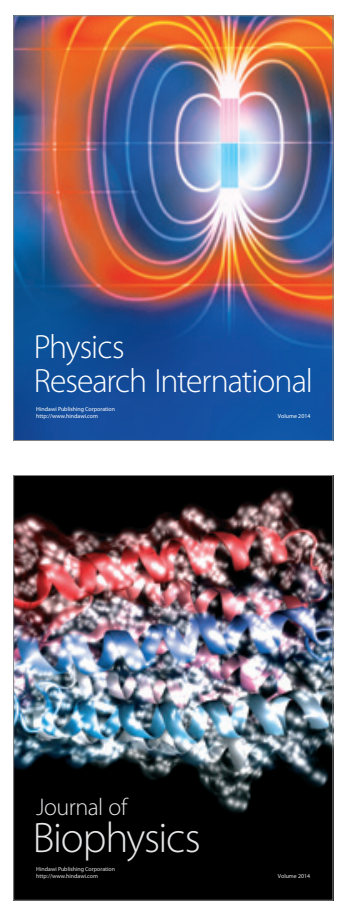
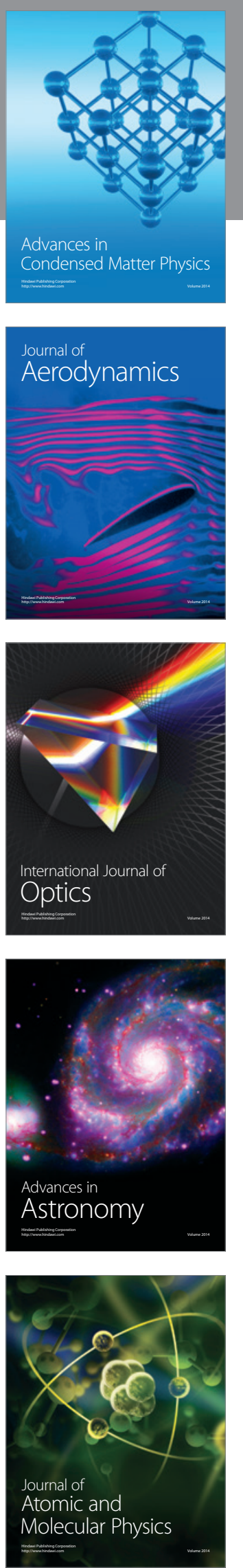